
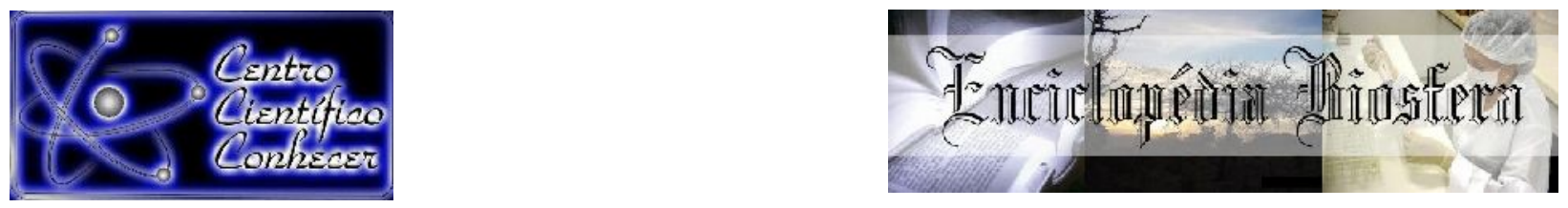

\title{
INTERPRETAÇÃO DE IMAGENS DE VEÍCULOS AÉREOS NÃO TRIPULADOS PARA AVALIAÇÃO DA SOBREVIVÊNCIA DE MUDAS EM PLANTIOS FLORESTAIS
}

\author{
Naiany Bonamichi Silva ${ }^{1}$, Ana Paula Dalla Corte ${ }^{1}$, Luani Rosa de Oliveira Piva ${ }^{1}$ \\ Carlos Roberto Sanquetta ${ }^{1}$ \\ ${ }^{1}$ Universidade Federal do Paraná, Curitiba, Brasil \\ E-mail: naianybonamichii@gmail.com
}

Recebido em: 06/04/2018 - Aprovado em: 10/06/2018 - Publicado em: 20/06/2018 DOI: 10.18677/EnciBio_2018A56

\begin{abstract}
RESUMO
Este trabalho teve como objetivo testar o desempenho de dois softwares para a detecção automática de sobrevivência de mudas de Eucalipto no Estado do Paraná. As fotografias aéreas foram obtidas a partir de um VANT (Veículo Aéreo Não-Tripulado) equipado com câmera RGB. Todo o processamento de geração da ortofoto foi executado com o software Postflight Terra 3D. Foram testados dois tratamentos: T1, detecção com software Spring; e T2, detecção com software eCognition. Para a comparação dos resultados adotou-se como testemunha (T0) a interpretação visual das imagens. Como resultado, obtiveram-se as seguintes classificações: a) indivíduos corretamente identificados; b) junção de dois ou mais indivíduos no mesmo polígono; c) omissão de indivíduos; d) área de copa por talhão. Os reconhecimentos dos indivíduos em ambos os softwares subestimaram o número real obtido em T0. Em T1, obteve-se omissão média de mudas de 6,9\% e, em T2, de 10,2\%. Ambos os tratamentos apresentaram classificação não correspondente de polígonos em suas classes, além de junção de duas ou mais árvores. T1 obteve média de junção de dois indivíduos de 3,1\% e T2 de 1,2\%. A média de classificação equivocada em T1 foi de $0,72 \%$, e de $0,14 \%$ em T2. A porcentagem de área de copas acompanhou o tamanho de cada talhão tanto em T1 como em T2. A substituição de métodos tradicionais de inventário de sobrevivência de mudas pela avaliação a partir de imagens VANT pode ser realizada, desde que consideradas as limitações dos erros observados, que ficaram entre 6,9 e $10,2 \%$.
\end{abstract}

PALAVRAS-CHAVE: detecção automática, programa computacional, vant

\section{INTERPRETATION OF IMAGES FROM UNMANNED AERIAL VEHICLES FOR SURVIVAL EVALUATION OF SEEDLINGS IN FOREST PLANTATIONS}

\begin{abstract}
The aim of this study was to test the performance of two software in automatic detection of Eucalyptus seedlings survival in Paraná State. The aerial photographs were obtained using the UAV eBee-Ag equipped with RGB camera. All orthophotos generation processing was executed with software Postflight Earth 3D. Two treatments were tested: T1, detection using the Spring software; and T2,
\end{abstract}


detection using the eCognition software. To results comparison, a parameter treatment (TO), with visual interpretation, was considered. The following classifications were obtained: a) individuals correctly determined; b) junction of two or more individuals in the same polygon; c) omission of individuals; d) crown area per stand. The individual recognition in both software underestimated the number obtained in T0. T1 presented $6.9 \%$ of average omission and T2 presented $10.2 \%$ of average omission. Both treatments presented non-correspondent classification of polygons in their classes, as well as the junction of two or more trees. T1 presented, by mean, the junction of two individuals of $3.1 \%$, and $\mathrm{T} 2,1.2 \%$. The mean of misclassification in T1 was $0.72 \%$, and $0.14 \%$ in T2. The percentage of crown area followed the size of each stand, both in T1 and T2. The substitution of traditional methods of seedling survival inventory by the evaluation of UAV images can be performed, considering the limitations of the observed errors, which were between 6.9 and $10.2 \%$.

KEYWORDS: automatic detection, software, UAV

\section{INTRODUÇÃO}

A aplicação de geotecnologias vem a cada dia se mostrando uma excelente ferramenta para o planejamento e obtenção de informações espaciais (PIROVANI et al., 2014; SANTOS et al., 2016). Essa tecnologia também possui grande potencial na área florestal, como, por exemplo, no suporte de inventários florestais tradicionais, pois dados espaciais obtidos podem conferir informações sobre o crescimento da floresta, dando suporte a decisões de gerenciamento de povoamentos florestais (VIANA et al., 2012; WALLACE et al., 2012).

Neste sentido, os VANTs se apresentam como uma ferramenta para otimização de avaliações e sistematização da metodologia de avaliação (GALIZIA et al., 2014). O uso dessa tecnologia constitui-se um importante meio para a obtenção de imagens em locais de difícil acesso (WHITEHEAD et al., 2014), oferecendo informações úteis para tomada de decisões. Além disso, a interpretação das imagens aéreas obtidas por meio de VANTs podem ser utilizados na área florestal para alguns objetivos, como monitorar a vegetação e sua condição de saúde, análise de terreno para hidrologia e cálculo volumétrico de biomassa, mapeamento de densidade e mapeamento de ervas daninhas além da vantagem de se poder usar as fotografias aéreas para observar cada estrutura de árvores individuais (ROKHMANA, 2015).

Atualmente, há uma ampla oferta de VANTs no mercado, com diferentes características de tamanho, autonomia de voo e câmeras. Entre os sensores mais utilizados nesse tipo de plataforma estão as câmeras fotográficas de baixo custo (HAUBECK; PRINZ, 2013). As facilidades existentes com a realização de voos autônomos remetem, principalmente, à diminuição de gastos por conta de não necessitarem de profissionais embarcados e, consequentemente, não oferecerem risco a tripulação (operador de câmera ou ao piloto), podendo assim ser utilizado em voos sobre áreas inacessíveis (TORRES-SÁNCHEZ et al., 2013). Além disso, a possibilidade de sobrevoo em baixas altitudes, a captura de imagens de alta resolução, o baixo custo de aquisição das imagens e a flexibilidade para o planejamento de voos são exemplos de outros atrativos desta tecnologia (PEÑA et al., 2015; TORRES-SÁNCHEZ et al., 2015).

A utilização de veículos aéreos não tripulados para coleta de dados aéreos pode ainda servir de instrumento para o desenvolvimento de novas plataformas para a coleta de imagens de alta resolução (DASH et al., 2017). 
Nesta perspectiva, a facilidade de obtenção de informações e, consequentemente, aumento de eficiência e automação dos processos a partir dos VANTs vem expandindo cada vez mais seu uso na área florestal como ferramenta auxiliar na silvicultura de precisão (CHAVES et al., 2015), sendo empregada, por exemplo, nas atividades de monitoramento e avaliação de falhas e sobrevivências de mudas em plantios florestais (MELLO et al., 2009).

Considerando o exposto, no presente trabalho foi abordado o potencial de uso das imagens captadas por VANTs para a realização de monitoramento de sobrevivência de mudas em plantios florestais, testando o desempenho de dois softwares (eCognition e Spring) na segmentação e classificação de imagens. Ainda, realizou-se o cálculo da porcentagem de sobrevivência de mudas de cada talhão, da porcentagem de classificação de copas, bem como da área das copas.

\section{MATERIAL E MÉTODOS}

\section{Área de estudo}

O estudo foi conduzido na Fazenda Mortandade, localizada no município de Telêmaco Borba-PR, a 249 km de Curitiba. Analisaram-se imagens obtidas a partir de voo realizado sobre quatro talhões com híbridos de Eucalyptus grandis $\mathrm{x}$ Eucalyptus urophylla, com plantio realizado em 21 de fevereiro de 2014, com espaçamento de 3,75 x 2,4 metros, totalizando cerca de 1.111 mudas por hectare. A área total dos quatro talhões é de 9,42 hectares, sendo que a área respectiva dos talhões 1, 2, 3 e 4 são 1,77 ha, 2,04 ha, 1,31 ha e 4,30 ha.

O município de Telêmaco Borba se caracteriza com o tipo climático Cfa, presente no lado oeste e litoral do estado e se caracteriza, conforme a classificação de Köppen, como clima oceânico úmido, apresentando temperatura no mês mais frio variando de $-3^{\circ} \mathrm{C}$ a $18^{\circ} \mathrm{C}$ e temperatura no mês mais quente superior a $22^{\circ} \mathrm{C}$, com verões quentes e precipitação mensal superior a $40 \mathrm{~mm}$ (ALVARES et al., 2013).

\section{Coleta e processamento de imagem VANT}

As fotografias aéreas foram obtidas em setembro de 2015 utilizando-se 0 VANT eBee-Ag, comercializado pela empresa SenseFly. De acordo com informações do fabricante, esse modelo de VANT possui hélice de propulsão elétrica, $96 \mathrm{~cm}$ de envergadura, peso de decolagem de $0,69 \mathrm{~kg}$ e autonomia máxima de 50 minutos de voo, permitindo cobrir uma área de até $12 \mathrm{~km}^{2}$. O VANT foi equipado com uma câmera RGB da marca Canon® (S110 RGB), que tem por caraterísticas $12 \mathrm{Mb}$ de resolução, sensor de 7,44 x 5,58 mm e distância focal de 4,37 m. Foram tomadas 41 fotografias aéreas para recobrimento dos quatro talhões. Todo o processamento pós voo para a geração da ortofoto foi executado com o software Postflight Terra 3D, sendo que as mesmas foram cedidas já processadas.

\section{Tratamentos}

Para a análise dos resultados adotou-se como testemunha (T0) a interpretação visual em tela das imagens das copas das mudas, com a respectiva contagem manual de cobertura, cujo cálculo das áreas foi realizado no software ArcGis, versão 10.4. Em seguida, testou-se dois tratamentos, sendo esses: a. tratamento $T 1$, no qual realizou-se o processamento das imagens no software Spring (versão 5.4.3); e b. tratamento T2, no qual o processamento das imagens 
foi realizado no software eCognition (versão 8.0). Em ambos os softwares foram realizados, para cada talhão, procedimentos de segmentação e classificação das imagens, visando a avaliação do percentual de acerto na identificação e nas áreas das copas identificadas.

No tratamento $\mathrm{T} 1$ as bandas utilizadas para o processamento das imagens foram as RGB, cujos comprimentos de onda são 0,64-0,67 $\mu \mathrm{m}, 0,53-0,59$ $\mu \mathrm{m}$ e 0,45-0,51 $\mu \mathrm{m}$ respectivamente. Como parâmetros de definição para o processo de segmentação foram utilizados os valores de limiar de similaridade igual à $4 \mathrm{e}$ área do pixel igual à 9 . Posteriormente à segmentação foi realizada a classificação em duas classes temáticas, a partir de amostras de treinamento, visando a determinação dos indivíduos/árvore sobreviventes. As duas classes temáticas foram:1) copas e 2) ausência de copas. O Spring adota o algoritmo de segmentação do tipo crescimento por regiões, do qual se utilizada das propriedades similares dos pixels. Para a classificação foi utilizado o classificador por região orientada a objeto do tipo supervisionada com classificador Bhattacharya.

No tratamento T2 foram empregadas para processamento as bandas RGB, com os mesmos comprimentos de ondas utilizados em T1. Para a segmentação foi utilizado algoritmo de segmentação multiresolution segmentation. O valor fixado para o parâmetro de fator de escala foi 10 e para os parâmetros peso para cada uma das bandas (peso para cor, peso para compacidade, forma e suavidade) mantiveram-se constantes no qual, sendo que os valores foram $1 ; 0,9 ; 0,5 ; 0,1$ e 0,5 respectivamente. Posteriormente, para a classificação das imagens, foram coletadas amostras de treinamento e criadas classes temáticas análogas às criadas no tratamento $T 1$, visando o agrupamento dos polígonos em suas categorias correspondentes. $\mathrm{O}$ eCognition utiliza $\mathrm{O}$ classificador por região orientada a objeto do tipo supervisionada.

\section{Análise dos dados}

A comparação dos tratamentos com a testemunha, para a identificação dos indivíduos e delimitação de suas respectivas copas dos polígonos, possibilitou avaliar o desempenho dos mesmos de acordo com os seguintes critérios:

a) indivíduos corretamente identificados;

b) junção de dois ou mais indivíduos no mesmo polígono;

c) omissão de indivíduos;

Além das variáveis relacionadas à classificação dos indivíduos, calculouse a porcentagem de área/copa por talhão como meio de comparação entre os tratamentos e a testemunha. Para verificar a aderência o número de copas contabilizadas nos tratamentos T1 e T2 com relação à T0, foi aplicado o teste não paramétrico Qui-quadrado, a um nível de $5 \%$ de probabilidade.

$$
\chi^{2}=\sum_{i=1}^{k} \frac{\left(f_{i}-\hat{f}_{i}\right)^{2}}{\hat{f}_{i}}
$$


Em que: $\chi^{2}=$ Qui-quadrado calculado; $f_{i}=$ frequência observada na i-ésima classe de diâmetro; $\hat{f}_{i}=$ frequência estimada na i-ésima classe de diâmetro; $k=$ número de classes de diâmetro.

A comparação entre o Qui-quadrado calculado $\left(\chi^{2}\right)$ e o Qui-quadrado tabelado $\left(\chi_{t a b}^{2}\right)$ foi realizada, sendo a hipótese $\mathrm{H}_{0}$ que $\chi^{2}=\chi^{2}{ }_{t a b}$. Caso o valor de $\chi^{2}$ seja menor que $\chi_{\text {tab }}^{2}$, considera-se uma não significância, ou seja, de que há aderência entre os dados.

Os erros de comissão, omissão e junção de copas presentes nos tratamentos T1 e T2 foram determinados manualmente, a partir de matriz de confusão, sendo que a comparação dos valores com relação à T0 foi realizada.

Para verificar a ocorrência de diferença estatística entre os tratamentos, foi aplicado um teste t pareado, a um nível de $5 \%$ de probabilidade, realizado no software R Studio, versão 1.1.442.

\section{RESULTADOS E DISCUSSÕES}

No tratamento testemunha (T0) contabilizou-se para os talhões 1, 2, 3 e 4 $1.787,2.170,1.307$ e 4.648 indivíduos, respectivamente, totalizando 9.912 indivíduos (Tabela 1). No tratamento T0, mais indivíduos foram contabilizados quando comparado aos tratamentos $\mathrm{T} 1$ e T2, os quais, entre si, apresentaram valores similares de indivíduos para todos os talhões avaliados.

TABELA 1. Quantificação do número de indivíduos dos 4 talhões localizados na fazenda Mortandade em Telêmaco Borba - PR.

\begin{tabular}{ccccccc}
\hline & \multicolumn{3}{c}{ Número de indivíduos } & \multicolumn{2}{c}{$\chi^{2}$ calculado } \\
\hline Talhão & $\begin{array}{c}\text { Plantio } \\
\text { inicial }\end{array}$ & T0 & T1 & T2 & T0-T1 & T0-T2 \\
\hline $\begin{array}{c}\text { Talhão 1 } \\
(1,77 \text { ha) }\end{array}$ & 1.966 & 1.787 & 1.673 & 1.606 & 7,768 & 20,399 \\
\hline $\begin{array}{c}\text { Talhão 2 } \\
(2,04 \text { ha) }\end{array}$ & 2.266 & 2.170 & 2.087 & 1.830 & 3,301 & 63,196 \\
\hline $\begin{array}{c}\text { Talhão 3 } \\
(1,31 \text { ha) }\end{array}$ & 1.451 & 1.307 & 1.139 & 1.195 & 24,780 & 10,497 \\
\hline $\begin{array}{c}\text { Talhão 4 } \\
(4,30 \text { ha) }\end{array}$ & 4.777 & 4.648 & 4.435 & 4.288 & 10,230 & 30,224 \\
\hline$\Sigma$ & - & - & - & - & 46,078 & 124,289 \\
\hline
\end{tabular}

Em que: $\Sigma=$ somatório; $\chi^{2}$ calculado= Qui-quadrado entre os tratamentos; $\chi^{2}$ tabelado $=0,35185$.

O teste de Qui-quadrado apontou diferença significativa entre os resultados de T0-T1, T0-T2, pois a somatória dos valores de Qui-quadrado calculados, para ambos os casos, foram maiores do que o valor tabelado. Dessa maneira, constatou-se que, estatisticamente, não houve aderência dos valores dos tratamentos $\mathrm{T} 1$ e T2 com o tratamento T0.

Em T1 o talhão com maior percentual de indivíduos não marcados foi o talhão 3, com omissão de 168 indivíduos, correspondente a 12,85\%. Nos talhões 1,2 e 4 houve erro de omissão iguais a 6,38\%, 3,82\% e 4,58\%, respectivamente. $\mathrm{Na}$ média dos quatro talhões houve a omissão de $6,9 \%$ do total de copas apresentado em T0. O talhão 4 apresentou maiores valores de erros de junção de copas, valores elevados aos comparados, por exemplo, ao talhão 3, com menores erros de junção. 
TABELA 2. Quantificação de erro de comissão e junção de copas no tratamento T1

\begin{tabular}{llcccc}
\hline \multirow{2}{*}{ Tratamento } & Palhão 1 & Talhão 2 & Talhão 3 & Talhão 4 \\
\cline { 2 - 6 } & Área total (ha) & 1,77 & 2,04 & 1,31 & 4,30 \\
\hline \multirow{3}{*}{ Tratamento 1 } & Erro de comissão $\left(\mathrm{m}^{2}\right)$ & 93,87 & 178,24 & 9,79 & 599 \\
\cline { 2 - 6 } & Erro/área (\%) & 0,53 & 0,87 & 0,07 & 1,39 \\
\cline { 2 - 6 } & Junção de 2 copas & 60 & 93 & 5 & 200 \\
\cline { 2 - 6 } & Junção de 3 copas & 6 & 19 & 0 & 28 \\
\cline { 2 - 6 } & Junção de 4 ou mais copas & 1 & 10 & 0 & 6 \\
\hline
\end{tabular}

No tratamento T2 o talhão 2 apresentou maior percentual de omissão, sendo $15,67 \%$, correspondente a 340 indivíduos (Tabela 3 ). Os talhões 1, 3 e 4 obtiveram média de omissão respectiva de $10,13 \%, 8,57 \%$ e $7,75 \%$. Na média dos talhões o tratamento não identificou $10,53 \%$ das copas. O maior percentual de junção de dois indivíduos foi visto no talhão 1 , com junção de $2,69 \%$. Os talhões 2, 3 e 4 apresentaram média de junção de dois indivíduos de $0,74 \%$, $0,23 \%$ e 1,29\% respectivamente. A junção de 3 e 4 indivíduos para os talhões 1 , 2 , 3 e 4 resultou em médias de $0,12 \%$ e $0,02 \%$, respectivamente. A maior média de erro de comissão foi obtida no talhão 1 , com $0,32 \%$ da área classificada de modo equivocado, a média dos 4 talhões foi de $0,14 \%$.

TABELA 3. Quantificação de erro de comissão e junção de copas no tratamento T2

\begin{tabular}{|c|c|c|c|c|c|}
\hline \multirow{2}{*}{ Tratamento } & Parâmetros & Talhão 1 & Talhão 2 & Talhão 3 & Talhão 4 \\
\hline & Área total (ha) & 1,77 & 2,04 & 1,31 & 4,30 \\
\hline \multirow{5}{*}{ Tratamento 2} & Erro de comissão $\left(\mathrm{m}^{2}\right)$ & 57,22 & 31,14 & 5,06 & 23,00 \\
\hline & Erro/área (\%) & 0,32 & 0,15 & 0,04 & 0,05 \\
\hline & Junção de 2 copas & 48 & 16 & 3 & 60 \\
\hline & Junção de 3 copas & 5 & 2 & 0 & 5 \\
\hline & $\begin{array}{l}\text { Junção de } 4 \text { ou mais } \\
\text { copas }\end{array}$ & 1 & 0 & 0 & 1 \\
\hline
\end{tabular}

Verificou-se que os reconhecimentos de copas em ambos os tratamentos (T1 e T2) subestimaram o número real obtido por contagem manual (T0), mostrando diferença significativa de resultados. Além disso, ambos apresentaram problemas de classificação de polígonos em suas classes não correspondentes e de junção de duas ou mais árvores, sendo a junção de dois indivíduos a mais frequente. No tratamento T1 os maiores percentuais de junção de duas copas foram obtidos nos talhões 2 e 4 com $4,30 \%$ das copas apresentando junção de dois indivíduos, no talhão 1 e 3 o percentual de junção foi de 3,36\% e 0,38\% respectivamente. A média de junção de dois indivíduos no tratamento foi de $3,09 \%$. A junção de 3 e 4 indivíduos para os talhões 1, 2, 3 e 4 foi, em média, de $0,46 \%$ e $0,16 \%$. Quanto ao erro de comissão, no tratamento T2 a maior média foi observada no talhão 4 , com 1,39\% da área identificada de modo incorreto (Tabela 2).

Comparando os resultados obtidos nos tratamentos T1 e T2 com o trabalho de Favarin et al. (2013), cuja omissão contabilizada foi de aproximadamente $10,11 \%$ em plantio comercial, os mesmos foram satisfatórios, uma vez que os resultados se mantiveram próximos ou abaixo dos resultados encontrados pelos autores. Os valores calculados obtidos no teste de Quiquadrado de erro de comissão e de junção de copas entre T1 e T2 mostraram-se ENCICLOPÉDIA BIOSFERA, Centro Científico Conhecer - Goiânia, v. 15 n.27; p. 613 2018 
acima do tabelado, ou seja, há diferença significativa em todas as comparações (Tabela 4).

Em ambos os tratamentos o agrupamento de copas se deu principalmente onde havia a presença de vegetação ao entorno das mudas, indicando dois ou mais indivíduos como apenas um indivíduo, motivo esse que pode ter causado também a identificação do solo como copas, o que é confirmado pdr Araújo et al. (2006) e Forre-Sanchéz et al. (2013), que afirmam que pode ocorrer agrupamento em povoamentos novos, principalmente se observada a presença de algum tipo de vegetação no entorno das mudas, como, por exemplo, vegetação mato-competidora (Figura 1).
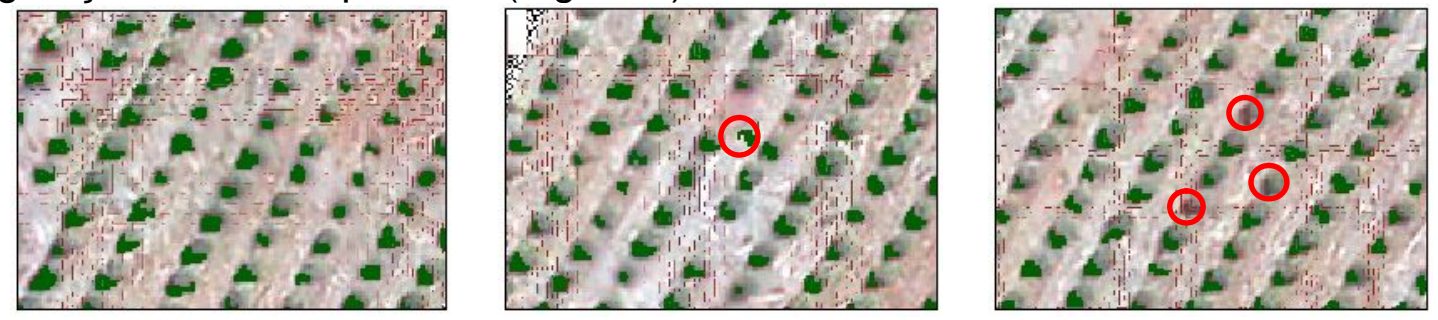

FIGURA 1. Falha de agrupamento dos polígonos em suas respectivas classes. Em ordem: 1) identificação correta dos indivíduos 2) identificação de copas não existente 3) não identificação de copa existente (fonte: Autores, 2017).

Erros de classificação nas áreas de bordadura também foram observados, podendo ser explicados pela alteração das características de parte da copa devido à sua irregularidade, que varia de indivíduo para indivíduo e quando há presença de sombra, visto que este tipo de problema pode ser observado próximo a regiões com sombra (SOUSA et al., 2010).

Os mesmos podem ser minimizados testando em $\mathrm{T} 1$ valores menores para o fator similaridade e maiores para o fator área pixels como visto no trabalho de Teodoro et al. (2014), onde se obteve melhores resultados de classificação. Espindola et al. (2007) ainda citam que valores muito baixos para o fator similaridade pode ocasionar subsegmentação, enquanto valores muito altos podem causar sobresegmentação, fato este que podem afetar diretamente as fases subsequentes (BAATZ; SCHAEPE, 2000).

Em T2 os erros podem ser minimizados quando testados valores distintos para forma, suavidade e compacidade além do fator escala, no trabalho de Nunes et al. (2014) usou-se para os parâmetros escala, forma e compacidade respectivamente $15,0,1$ e 0,5 . Em ambos os tratamentos deve-se coletar um número suficiente de amostras e que as mesmas sejam representativas em relação às classes estabelecidas (LU; WENG, 2007).

Outro fator que ocasiona erros é a pouca nitidez em algumas regiões da foto, bem como a semelhança das características espectrais, que se referem à composição de cores dos alvos, em alguns pontos da foto podem fazer com que as mudas não sejam destacadas, subestimando os resultados, evento este que pode ser minimizado realizando a coleta de dados antes, em média aos 6 meses, onde além da sobrevivência do plantio pode-se coletar dados da qualidade silvicultural do mesmo (ARAÚJO et al., 2006).

No cálculo automático de superfície de copa, ambos os tratamentos acompanharam a área/copas de acordo com o tamanho da área, sendo o talhão 4 com maior área, seguido do talhão 2, 1 e 3 (Tabela 4). 
TABELA 4. Porcentagem de área/copas total e área de copa individual obtidas nos tratamentos T0, T1 e T2.

\begin{tabular}{|c|c|c|c|c|c|}
\hline \multirow{2}{*}{ Tratamento } & Parâmetro & Talhão 1 & Talhão 2 & Talhão 3 & Talhão 4 \\
\hline & Área total (ha) & 1,77 & 2,04 & 1,31 & 4,30 \\
\hline \multirow{3}{*}{ Tratamento 0} & Área copa (ha) & 0,159 & 0,16 & 1,10 & 0,50 \\
\hline & Área copa (\%) & 8,96 & 7,89 & 7,60 & 11,66 \\
\hline & Área individual $\left(\mathrm{m}^{2}\right)$ & 0,89 & 0,74 & 0,76 & 1,08 \\
\hline \multirow{3}{*}{ Tratamento 1} & Área copa (ha) & 0,19 & 0,25 & 0,11 & 0,66 \\
\hline & Área copa (\%) & 10,68 & 12,16 & 8,09 & 15,23 \\
\hline & Área individual $\left(\mathrm{m}^{2}\right)$ & 1,13 & 1,19 & 0,93 & 1,48 \\
\hline \multirow{3}{*}{ Tratamento 2} & Área copa (ha) & 0,13 & 0,16 & 0,092 & 0,48 \\
\hline & Área copa (\%) & 7,12 & 7,75 & 7,02 & 11,05 \\
\hline & Área individual $\left(\mathrm{m}^{2}\right)$ & 0,78 & 0,86 & 0,77 & 1,11 \\
\hline
\end{tabular}

As porcentagens de copa em T0 variaram de 7,60\% a 11,66\%, em T1 de $8,09 \%$ a $15,23 \%$ e em T2 de $7,02 \%$ a $11,05 \%$, os resultados mostram-se condizentes, uma vez que Nunes et al. (2014) obtiveram porcentagem média de copas variando de $15,2 \%$ a 20,3\% em plantios de Eucalyptus urograndis aos seis anos.

No tratamento T1 obteve-se erro percentual máximo de $54,12 \%$ enquanto que no tratamento T2 o percentual máximo encontrado foi de 20,53\%. Comparando os resultados obtidos com o resultado de $36,9 \%$ encontrado no trabalho de Soares (2015), o tratamento T1 mostrou-se acima do erro percentual encontrado pelo autor e T2 se manteve abaixo do resultado.

Em T1 houve superestimativa dos resultados em todos os talhões, fato este que pode ser justificado pela classificação do mesmo ter obtido grande quantidade de junções de indivíduos e por ter apresentado uma aproximação maior do número de indivíduos de T0. Em T2 todas as superfícies de copa obtidas ficaram abaixo da superfície de copa de T0, sendo justificada por ter sempre subestimado os resultados de número de indivíduos e por apresentar menor quantidade de junções (2, 3 ou 4 indivíduos) que T1.

A aplicação do teste t pareado, que visou comparar a diferença entre os tratamentos T1 e T2, resultou em um valor de p igual a 0,2139, maior que o valor de probabilidade estipulado $(0,2139>0,05)$. Portanto, não houve diferença entre os valores de copas contabilizadas nos dois tratamentos, apontando que a detecção de indivíduos com o software Spring não diferiu, estatisticamente, da detecção realizada a partir do eCognition.

\section{CONCLUSÃO}

Os resultados mostraram que as eficiências do uso dos softwares no reconhecimento de mudas de eucalipto não se diferiram estatisticamente. Entretanto, tendo em vista que T2, que consistiu no uso do software eCognition, apresentou menores erros de comissão, omissão e junção de duas ou mais copas, recomenda-se a utilização do mesmo na detecção automática de mudas de Eucalipto.

A substituição de métodos tradicionais de inventário de sobrevivência por imagens VANT, classificadas com os softwares Spring e eCognition, pode ser realizada desde que observadas as limitações que giram entre $6,9-10,2 \%$ de erro se $o$ processamento se der sem nenhuma edição. Caso exista a possibilidade de 
edição para a correção de erros aqui relatados, os erros do inventário de sobrevivência poderão ser minimizados em ambos os tratamentos.

Sugere-se que, para minimização de erros, pode-se abordar valores distintos para os parâmetros que influenciam na segmentação em ambos os tratamentos, buscando maior adequação de acordo com o tipo de dado a ser analisado e finalidade dos resultados, melhorando o processo subsequente de classificação e evitando esforço de edições subsequentes.

Sugere-se ainda testar alternativas de combinação de parâmetros técnicos com a finalidade de melhorar o resultado, como por exemplo, a inclusão de índices de vegetação na segmentação e classificação.

\section{AGRADECIMENTOS}

Agradecimentos a empresa Klabin S.A pela disponibilização dos dados, ao Centro de Excelência em Pesquisas sobre Fixação de Carbono na Biomassa (BIOFIX) pela estrutura disponibilizada para realização do trabalho, ao Conselho Nacional de Desenvolvimento Científico e Tecnológico (CNPq), pela disposição da bolsa iniciação cientifica e a todos os colegas que contribuíram para a realização desse trabalho.

\section{REFERÊNCIAS}

ALVARES, C. A.; STAPE, J. L.; SENTELHAS, P. C.; GONÇALVES, J. L. M.; SPAROVEK, G. Koppen's climate classification map for Brazil. Meteorologische Zeitschrift, v. 22, ํㅡ. 6, p. 711-728, 2013. DOI: 10.1127/0941-2948/2013/0507. Disponível em: <http://dx.doi.org/10.1127/0941-2948/2013/0507>. doi: 10.1127/0941-2948/2013/0507.

ARAÚJO, M. A.; CHAVIER, F.; DOMINGOS, J. L. Avaliação do potencial de produtos derivados de aeronaves não tripuladas na atividade florestal. Ambiência, v.2, p. 69-82, 2006. Disponível em: < https://revistas.unicentro.br/index.php/ambiencia/article/view/258/338>.

BAATZ, M.; SCHÃPE, A. Multiresolution Segmentation: an optimization approach for high quality multi-scale image segmentation. 2000. Angewandte Geographische Informations - Verarbeitung XII: 12-23. Disponível em: < http://www.ecognition.com/sites/default/files/405_baatz_fp_12.pdf>.

ChAVES, A. A.; LA SCALEA, R. A.; COlTURATO, A. B.; KAWABATA, C. L. O.; FURTADO, E. L.; BRANCO, K. R. L. J. C. Uso de VANTs e processamento digital de imagens para a quantificação de áreas de solo e de vegetação. In: Simpósio brasileiro de sensoriamento remoto, - SBSR, 2015, João Pessoa, Brasil. Disponível em: < http://www.dsr.inpe.br/sbsr2015/files/p1143.pdf>.

DASH, J. O.; WATT. M. S.; PEARSE, G. D.; HEAPHY, M.; DUNGEY, H.S. Assessing very high resolution UAV imagery for monitoring forest health during a simulated disease outbreak. ISPRS Journal of Photogrammetry and Remote Sensing, v.131, p. 1-14, 2017. Disponível em: <https://doi.org/10.1016/j.isprsjprs.2017.07.007>. doi: 10.1016/j.isprsjprs.2017.07.007.

ESPINDOLA, G.; CAMARA, G.; REIS, I.; BINS, L.; MONTEIRO, A. Parameter 
selection for region-growing image segmentation algorithms using spatial autocorrelation. International Journal of Remote Sensing. v. 27, p. 3035-3040, 2007. Disponível em: <https://doi.org/10.1080/01431160600617194>. doi: 10.1080/01431160600617194.

FAVARIN, J. A. S.; PEREIRA, R. S.; PEGORARO, A. J.; LIPPERT; D. B. Obtenção de fotografias aéreas de um povoamento de Pinus taeda $\mathrm{L}$. com o VANT Microdrone MD4-1000. In: Simpósio brasileiro de sensoriamento remoto - SBSR, 2013, Foz do Iguaçu, Brasil. Disponível em: < http://marte2.sid.inpe.br/rep/dpi.inpe.br/marte2/2013/05.29.00.22.16>.

GALIZIA, L. F. C.; RAMIRO, G. A.; ROSA, C. J. C. Qualidade das atividades silviculturais e silvicultura de precisão. Série Técnica IPEF, v. 24, n. 45, 2016. Disponível em: < www.ipef.br/publicacoes/stecnica/nr45/st45-cap07.pdf>.

HAUBECK, K.; PRINZ, T. A; uav-based low-cost stereo camera system for archaeological surveys - experiences from doliche (Turkey). Int. Arch. Photogramm. Remote Sens. Spatial Inf. Sci., XL-1/W2, 195-200, 2013. Disponível em: <https://doi.org/10.5194/isprsarchives-XL-1-W2-195-2013>. doi: 10.5194/isprsarchives-XL-1-W2-195-2013.

LU, D.; WENG, Q. A survey of image classification methods and techniques for improving classification performance. International Journal of Remote Sensing, v. 28, n. 5, p. 823-870, 2007. Disponível em: <https://doi.org/10.1080/01431160600746456>. 10.1080/01431160600746456.

MELLO, J. M.; DINIZ, F. S.; OLIVEIRA, A. D. O.; MELLO, C. R.; SCOLFORO, J. R. S.; ACERBI JÚNIOR, F. W. Continuidade espacial para características dendrométricas (número de fustes e volume) em plantios de Eucalyptus grandis. Revista Árvore, Viçosa, v. 33, n. 1, p. 185-194, 2009. Disponível em: < http://dx.doi.org/10.1590/S0100-67622009000100020>. doi: 10.1590/S010067622009000100020.

NUNES, G. M.; VIEIRA, D. J. E.; CARVALHO, S. P. C. Avaliação preliminar de dados obtidos por VANT em talhão florestal clonal de Eucalyptus urograndis H13 no estado de Mato Grosso. In: XI Seminário de Atualização em Sensoriamento Remoto e Sistemas de Informações Geográficas aplicados à Engenharia Florestal, Curitiba. Anais. Paraná, PR: XI SenGeF, 2014, p. 211-218. Disponível em:

<http://www.ufmt.br/ufmt/unidade/userfiles/publicacoes/2adfeb89164b6b251be167 d82a47410b.pdf>.

PEÑA, J. M.; TORRES-SÁNCHEZ, J.; SERRANO-PÉREZ, A.; CASTRO, A. I.; LÓPEZ-GRANADOS, F. Quantifying efficacy and limits of unmanned aerial vehicle (UAV) technology for weed seedling detection as affected by sensor resolution. Sensors, v. 15, n. 3, p. 5609-5626, 2015. Disponível em: < https://doi.org/10.3390/s150305609>. doi:10.3390/s150305609. 
PIROVANI, D. B. et al. Análise espacial de fragmentos florestais na Bacia do Rio Itapemirim, ES. Revista Árvore, v. 38, n. 2, p. 271-281, 2014. Disponível em: <http://dx.doi.org/10.1590/S0100-67622014000200007>. doi: 10.1590/S010067622014000200007.

ROKHMANA, C.A. The Potential of UAV-based Remote Sensing for Supporting Precision Agriculture in Indonesia. Procedia Environmental Sciences, v.24, p. 245-253, 2015. Disponível em: <https://doi.org/10.1016/j.proenv.2015.03.032>. doi: 10.1016/j.proenv.2015.03.032.

SANTOS, A. R. et al. Influence of relief on permanent preservation areas. Science of the Total Environment, v. 541, p. 1296-1302, 2016. Disponível em: <https://doi.org/10.1016/j.scitotenv.2015.10.026>. 10.1016/j.scitotenv.2015.10.026.

SENSEFLY. O drone de mapeamento profissional. Disponível em: <https://www.sensefly.com/app/uploads/2017/10/web-AAFF-eBee-PT.pdf>. Acesso em: 12/09/2017.

SOARES, P; FIRMINO, P; TOMÉ, M; CAMPAGNOLO, M; OLIVEIRA, J et al. Utilização de veículos aéreos não tripulados no inventário florestal - 0 caso do montado de sobro. In: Conferência Nacional de Cartografia e Geodesia-CNCG, p. $1-8,2015.2$ Disponível em: < http://viiicncg.ordemengenheiros.pt/fotos/editor2/VIIICNCG/cncg2015_comunicao_ 45.pdf>.

SOUSA, A. M. O; GONÇALVES, A. C; SILVA, J. R. M; MESQUITA, P. Segmentação e classificação de tipologias florestais a partir de imagens Quickbird. Ambiência, v. 6, p. 57-66, 2010. Disponível em: < https://revistas.unicentro.br/index.php/ambiencia/article/view/873>.

TEODORO, A. C; ARAÚJO, R. Exploration of the OBIA methods available in SPRING noncommercial software to UAV data processing. SPIE Proceedings, v. 9245, p. 1-11, 2014. Disponível em: <https://doi.org/10.1117/12.2066468>. doi: 10.1117/12.2066468.

TORRES-SÁNCHEZ, J.; LÓPEZ-GRANADOS, F.; CASTRO, A. I.; PEÑABARRAGÁN, J. M. Configuration and specifications of an unmanned aerial vehicle (UAV) for early site specific weed management. PloS one, v. 8, n. 3, p. e58210, 2013. Disponível em: <https://doi.org/10.1371/journal.pone.0058210>. doi: 10.1371/journal.pone.0058210.

TORRES-SÁNCHES, J; LÓPEZ-GRANADOS, F; SERRANO, N; ARQUERO, O; PEÑA, J.M. High-throughput 3-D monitoring of agricultural-tree plantations with unmanned aerial vehicle (UAV) technology. Plos ONE, v. 10, n. 6, p. e0130479, 2015. Disponível em: <https://doi.org/10.1371/journal.pone.0130479>. doi: 10.1371/journal.pone.0130479.

VIANA, H.; ARANHA, J.; LOPES, D.; COHEN, W.B. Estimation of crown biomass of Pinus pinaster stands and shrubland above-ground biomass using forest 
inventory data, remotely sensed imagery and spatial prediction models. Ecological Modelling, v. 226, p. 22-35, 2012. Disponível em <http://dx.doi.org/10.1016/j.ecolmodel.2011.11.027>. doi: 10.1016/j.ecolmodel.2011.11.027.

WALLACE, L.; LUCIEER, A.; MALENOVSKÝ, Z.; TURNER, D.; VOPĚNKA, P. Assessment of forest structure using two UAV techniques: A comparison of airborne laser scanning and structure from motion (SfM) point clouds. Forests, v. 7, n. 3, p. 62, 2012. Disponível em: <http://dx.doi.org/10.3390/rs4061519>. doi: $10.3390 /$ rs 4061519 .

WHITEHEAD, K.; HUGENHOLTZ, C.H. Remote sensing of the environment with small unmanned aircraft systems (UASs), part 1: A review of progress and challenges. Journal of Unmanned Vehicle Systems, v. 2, n. 3, p. 69-85, 2014. Disponível em: <http://dx.doi.org/10.1139/juvs-2014-0007>. doi: 10.1139/juvs2014-0007. 